\title{
Responses of the Human Inner Ear to Low- Frequency Sound
}

\author{
Markus Drexl, Eike Krause, Robert Gürkov and Lutz Wiegrebe
}

\begin{abstract}
The perceptual insensitivity to low frequency (LF) sound in humans has led to an underestimation of the physiological impact of LF exposure on the inner ear. It is known, however, that intense, LF sound causes cyclic changes of indicators of inner ear function after LF stimulus offset, for which the term "Bounce" phenomenon has been coined.

Here, we show that the mechanical amplification of hair cells (OHCs) is significantly affected after the presentation of LF sound. First, we show the Bounce phenomenon in slow level changes of quadratic, but not cubic, distortion product otoacoustic emissions (DPOAEs). Second, Bouncing in response to LF sound is seen in slow, oscillating frequency and correlated level changes of spontaneous otoacoustic emissions (SOAEs). Surprisingly, LF sound can induce new SOAEs which can persist for tens of seconds. Further, we show that the Bounce persists under free-field conditions, i.e. without an in-ear probe occluding the auditory meatus. Finally, we show that the Bounce is affected by contralateral acoustic stimulation synchronised to the ipsilateral LF sound. These findings clearly demonstrate that the origin of the Bounce lies in the modulation of cochlear amplifier gain. We conclude that activity changes of OHCs are the source of the Bounce, most likely caused by a temporary disturbance of $\mathrm{OHC}$ calcium homeostasis. In the light of these findings, the effects of long-duration, anthropogenic LF sound on the human inner ear require further research.
\end{abstract}

\footnotetext{
M. Drexl $(\square) \cdot$ E. Krause $\cdot$ R. Gürkov

German Center for Vertigo and Balance Disorders (IFB), Department of Otorhinolaryngology, Head and Neck Surgery, Grosshadern Medical Centre, University of Munich, 81377 Munich, Germany

e-mail: markus.drexl@med.uni-muenchen.de

E. Krause

e-mail: eike.krause@med.uni-muenchen.de

R. Gürkov

e-mail: robert.guerkov@med.uni-muenchen.de

L. Wiegrebe

Division of Neurobiology, Dept. Biology II, University of Munich, 82152 Martinsried, Germany e-mail: lutzw@1mu.de 
Keywords Cochlea Calcium homeostasis $\cdot$ Cochlear amplifier ' Outer hair cells · Inner hair cells $\cdot$ Bounce phenomenon $\cdot$ Otoacoustic emissions

\section{Introduction}

For decades, low-frequency sound, i.e. sound with frequencies lower than $250 \mathrm{~Hz}$ (Berglund et al. 1996), has been considered to largely bypass the inner ear even at intense levels, simply because human hearing thresholds for frequencies below $250 \mathrm{~Hz}$ are relatively high. Recent evidence from animal models shows that physiological cochlear responses to LF sound are even larger than those evoked by equallevel, higher frequencies in the more sensitive range of hearing (Salt et al. 2013). No data for human subjects are available, but, considering the higher sensitivity of humans for LF sounds, similar results can be expected (Salt et al. 2013).

Hirsh and Ward (1952) observed temporary deteriorations of human absolute thresholds about 2 min after presenting subjects with an intense, non-traumatic LF tone. Later on, the term 'Bounce' was used to describe bimodal changes in absolute thresholds starting with a sensitisation period followed by an about equal-duration temporary desensitisation (Hughes 1954).

Perceptual thresholds essentially reflect the sensitivity of inner hair cells (IHCs), which are functionally coupled to inner ear fluids (Nowotny and Gummer 2006; Guinan 2012). IHCs are therefore sensitive to basilar-membrane velocity, which decreases with decreasing frequency. OHCs, in contrast, are mechanically linked to both the basilar membrane and the tectorial membrane. OHCs are therefore sensitive to basilar membrane displacement (Dallos et al. 1982; Dallos 1986), which does not decrease with decreasing stimulus frequency. Thus, $\mathrm{OHCs}$ are more sensitive to LF sound than IHCs and it is this difference in LF sensitivity, which contributes to the LF limit of sound perception (Salt and Hullar 2010; Salt et al. 2013). In humans, non-invasive recordings of DPOAEs allow indirect access to OHC function while SOAEs represent, for ears that exhibit them, a more direct and very sensitive marker of OHC function. SOAEs are narrowband acoustic signals which are spontaneously emitted by the inner ear in the absence of acoustic stimulation. Human SOAEs persist over years and are relatively stable in both frequency and level (Burns 2009).

Here, we use both DPOAE and SOAE measurements to assess LF-induced changes of cochlear physiology and active sound amplification. Specifically, we monitored the sound level and frequency of DPOAEs and SOAEs before and after the exposure to a $90 \mathrm{~s} \mathrm{LF}$ sinusoid with $30 \mathrm{~Hz}$ and a level of $80 \mathrm{dBA}(120 \mathrm{~dB}$ SPL). Both the sound level and the exposure duration were controlled to be within the exposure limits for normal working conditions as regulated by the European Commission Noise at Work Directive 2003/10/EC. 


\section{Methods}

Data were collected from young adult normal hearing subjects. The ethics committee of the University Hospital of the Ludwig-Maximilians University Munich, Germany, in agreement with the Code of Ethics of the World Medical Association (Declaration of Helsinki) for experiments involving humans, approved the procedures, and all subjects gave their written informed consent. An Etymotic Research 10C DPOAE probe system was used for recording of OAEs. The LF tone $(30 \mathrm{~Hz}$ sine wave, $120 \mathrm{~dB}$ SPL, $90 \mathrm{~s}$, including $0.1 \mathrm{~s}$ raised-cosine ramps) was supplied by a separate loudspeaker (Aurasound NSW1-205-8A). This loudspeaker was connected via a $50 \mathrm{~cm}$ polyethylene tube (inner diameter $1 \mathrm{~mm}$ ) and the foam ear tip of the ER-10C DPOAE probe so that it faced the tympanic membrane. The loudspeaker was driven by a Rotel RB-960BX power amplifier. Stimulation to the contralateral ear was provided by an Etymotic Research 4PT earphone, which was sealed into the contralateral ear canal with foam ear tips. The earphone was driven by the headphone amplifier of the audio interface (RME audio Fireface UC, fs $=44.1 \mathrm{kHz}$ ) which was programmed (using MatLab and the HörTech SoundMexPro audio toolbox) for synchronous stimulation and recording of all required inputs and outputs.

\section{Results}

\subsection{Effect of LF Sound Exposure on DPOAEs}

The effect of the $80 \mathrm{dBA}$ LF exposure on quadratic (QDP) and cubic (CDP) OAEs is shown in Fig. 1a and b, respectively. In 14 out of 20 tested subjects, the LF exposure induced a subsequent increase of the QDP level lasting for about 60 to $90 \mathrm{~s}$ (see Fig. 1a). QDP levels increased with a median of $3.4 \mathrm{~dB}$. In most cases, this QDP increase was followed by a similar QDP decrease (median: $-2.4 \mathrm{~dB}$ ), at about 120 $150 \mathrm{~s}$ post-exposure. This decrease slowly recovered to pre-exposure QDP levels. The median duration of the overall oscillatory change of the QDP level was $214 \mathrm{~s}$.

In many cases it was also possible to extract CDP levels from the same recording (albeit f2/f1 ratios were optimized to achieve maximum QDP levels). Typically, we observed no significant changes of CDP level after LF sound exposure (Fig. 1b).

\subsection{Effect of LF Sound Exposure on SOAEs}

We recorded 80 SOAEs from 27 ears of 16 young, normal-hearing subjects. The median SOAE sound levels were 0.6 dB SPL (first and third quartiles, $-4.5 \mathrm{~dB}$ $\mathrm{SPL}$; 4.0 dB SPL) with a signal-to-noise ratio of $16.6 \mathrm{~dB}(11.6 \mathrm{~dB}, 23.5 \mathrm{~dB})$. 
Fig. 1 Effect of LF sound exposure on quadratic distortion products (QDP, A, $N=28$ from 14 subjects) and cubic distortion products (CDP, $\mathrm{B}, N=22$ from 11 subjects). QDPs oscillate significantly after LF offset (a) whereas CDPs are rarely affected, $\mathbf{b}$ bold black lines represent median DPOAE measures, fine grey lines are individual DPOAE measures. (Modified after Drexl et al. (2014), with permission)
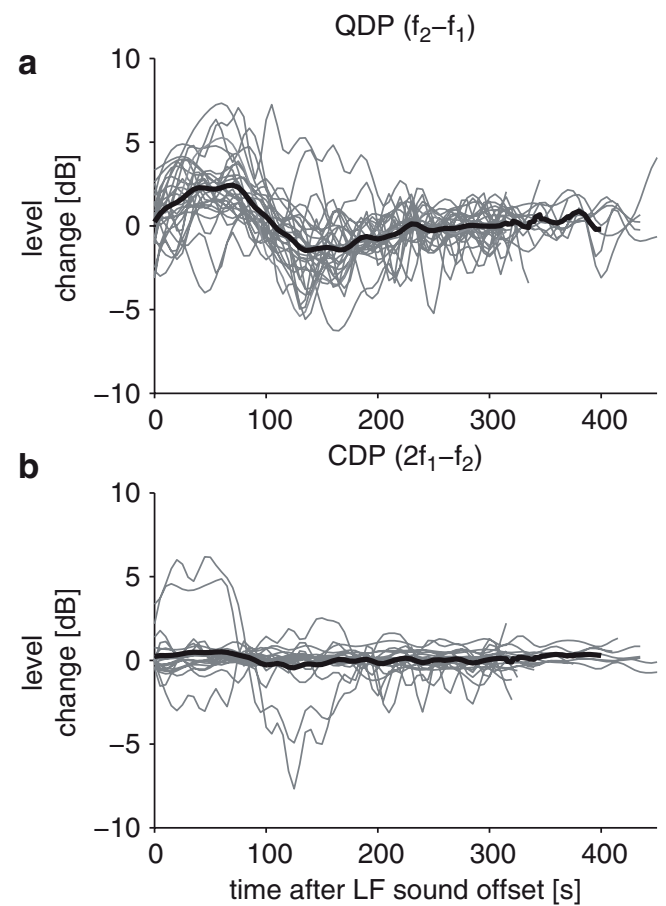

After LF sound stimulation, 56 of these 80 SOAEs increased in both sound level and frequency. This increase was followed by a decrease of both level and frequency relative to pre-exposure (see Fig. 2a). In 10 of the 80 pre-exposure SOAEs, we observed an inverted pattern with an initial level and frequency decrease, followed by a level and frequency increase.

SOAE level- and frequency oscillations were fitted with an (inverted-phase) underdamped sinusoidal oscillation. The period of the fitted sinusoid was $257 \mathrm{~s}$ $(202 \mathrm{~s}, 294 \mathrm{~s})$ for the level time course and $252 \mathrm{~s}(215 \mathrm{~s}, 367 \mathrm{~s})$ for the frequency time course. The time constant of the damped sinusoid for level changes was $120 \mathrm{~s}$ $(76 \mathrm{~s}, 157 \mathrm{~s})$ and for frequency changes $94 \mathrm{~s}(58 \mathrm{~s}, 141 \mathrm{~s})$. SOAE frequency changes amounted to 5 Cent (4 Cent, 9 Cent) with peak values of 25 Cent. Relative to the SOAE frequency in the control condition, the frequency showed initial maximum increases of 4 Cent (3 Cent, 7 Cent), followed by maximum decreases of 1 Cent (0 Cent, 2 Cent).

17 of 21 tested subjects revealed an overall of 56 new SOAEs, which had not been measurable before LF stimulation (see Fig. 2b). These new SOAEs were characterized by an initial level and frequency increase, qualitatively similar to the preexisting SOAEs. Comparable to the enhancing half cycle of Bouncing SOAEs, their level and frequency oscillated before they disappeared into the noise floor. The duration of the level and frequency changes was $67.5 \mathrm{~s}$ (47.5 s, $90 \mathrm{~s})$. New SOAEs started to arise within $12.5 \mathrm{~s}(5 \mathrm{~s}, 25 \mathrm{~s})$ after LF sound offset and reached a level maximum at $50 \mathrm{~s}(35 \mathrm{~s}, 62.5 \mathrm{~s})$ after LF offset. The maximum SOAE level was 

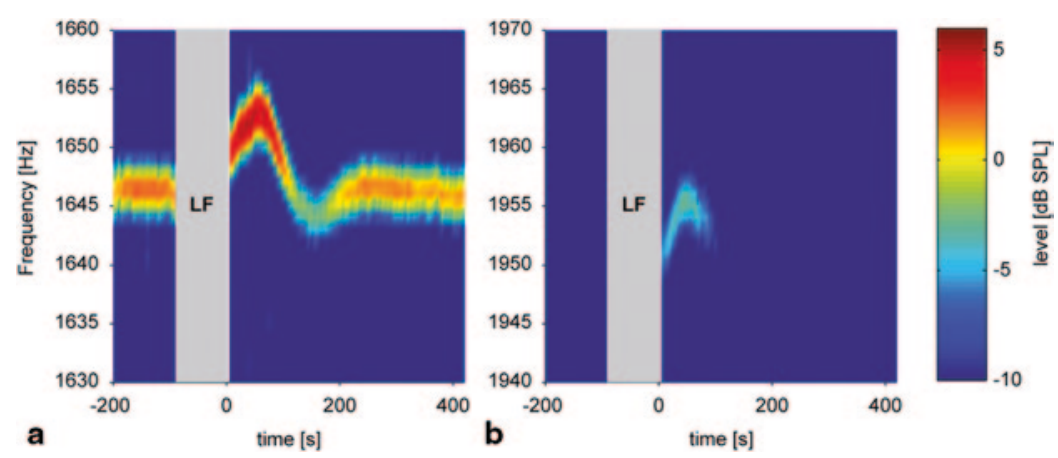

Fig. 2 a Exemplary level and frequency changes of an SOAE recorded pre- and post LF sound exposure. The grey bar indicates the presentation of the LF stimulus $(30 \mathrm{~Hz}, 80 \mathrm{dBA}, 90 \mathrm{~s})$. b Same as in a, but for a new SOAE which only appears for a short period after LF exposure. (Modified after Kugler et al. (2014), licensed under CC BY 4.0 (https://creativecommons.org/licenses/ by/4.0/))

$-0.3 \mathrm{~dB}$ SPL (-4.1 dB SPL, 4.9 dB SPL) with a signal to noise ratio of $13.8 \mathrm{~dB}$ $(11.9 \mathrm{~dB}, 17.6 \mathrm{~dB})$. The difference between the new SOAE frequency maximum and minimum was 4 Cent ( 1 Cent, 6 Cent). The time course of level and frequency changes was almost identical and maximum level and frequency changes coincided.

\subsection{SOAE Bouncing in the free Sound Field}

Although the observed pattern of synchronized SOAE frequency- and amplitude changes is incompatible with the SOAE bouncing being elicited by changes in middle ear impedance, it is conceivable that bouncing may be only seen in the closed sound field where the auditory meatus is blocked by the OAE probe. Here, we recorded SOAEs in the open meatus using an ER10C probe microphone fitted to the meatus via an about $8 \mathrm{~cm}$ silicon tube $(2.8 \mathrm{~mm}$ outer diameter) which did not block the meatus. The tip of the tube was positioned about $10 \mathrm{~mm}$ in front of the tympanum. LF exposure was provided by two powerful custom-made subwoofers. Subjects lay on a deck chair in a long basement corridor at a point where standing waves in the corridor maximised the sound level at $30 \mathrm{~Hz}$. LF exposure was $118 \mathrm{~dB}$ SPL for $90 \mathrm{~s}$. Photos of LF stimulation apparatus and probe-tube placement are shown in Fig. 3a, b.

Both ears of 45 young, normal-hearing subjects were screened for SOAEs. 33 subjects showed at least one SOAE in one ear. Overall we could record in the open meatus about $52 \%$ of those SOAEs detectable in a sound-attenuated room and a closed-ear recording technique. The remaining $48 \%$ were not significantly above the much higher noise floor of the free-field, open-meatus measurement.

Exemplary measurements of both permanent and transient SOAEs are shown in Fig. $3 c$ and $d$ in the same format as Fig. 2. Indeed many of those $48 \%$ SOAEs 

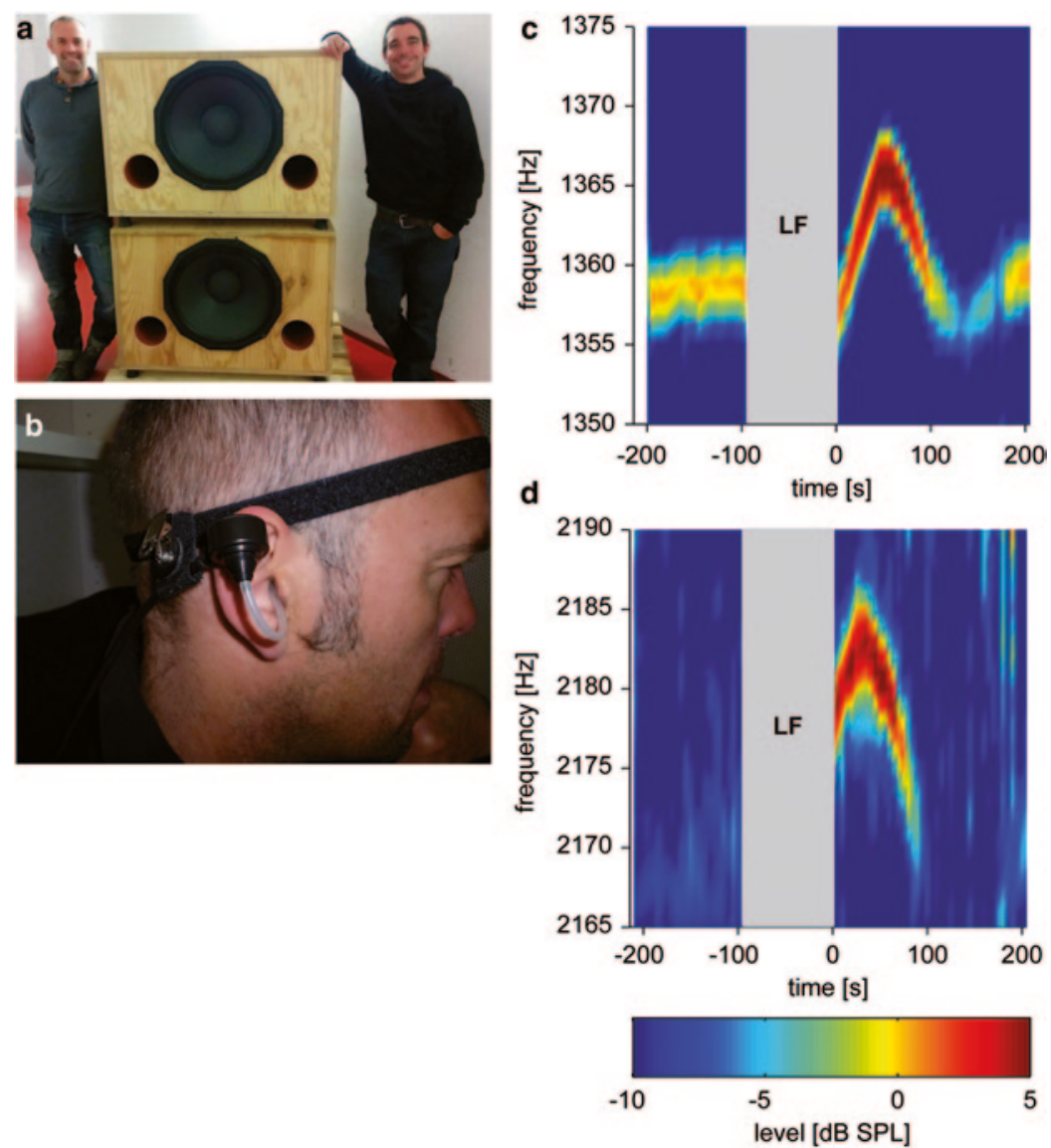

Fig. 3 LF free-field stimulation subwoofers (a), and positioning of the SOAE recording probe in the open ear canal (b), Exemplary recordings in the open meatus of a pre-existing SOAE (c), and a transient SOAE (d) after free-field exposure to a $118 \mathrm{~dB} \mathrm{SPL}, 30 \mathrm{~Hz}, 90 \mathrm{~s} \mathrm{LF}$ sound (grey bar)

that had been initially identified in the closed-meatus measurements, but could no longer be detected in the open meatus, appeared directly after the LF exposure for a short time period, before falling again below the noise floor (Fig. 3d). These data clearly show that Bouncing of SOAEs can indeed be elicited by free-field exposure to LF sound sources of natural or anthropogenic origin.

\subsection{Effect of Contralateral Acoustic Stimulation (CAS)}

Patuzzi (2011) suggested that large receptor-potentials elicited by low-frequency stimulation produce a net $\mathrm{Ca}^{2+}$ influx. The Bounce presumably reflects an underdamped, homeostatic readjustment of increased $\mathrm{Ca}^{2+}$ concentrations and related 
gain changes after low-frequency sound offset. Here, we tested this hypothesis by activating the medial olivocochlear (MOC) efferent system during presentation of the Bounce-evoking LF sound. The MOC system is known to modulate $\mathrm{OHC} \mathrm{Ca}^{2+}$ concentrations (Sridhar et al. 1997) and receptor potentials (Fex 1967) and therefore it should modulate the characteristics of the Bounce. Here, CAS was provided simultaneously to the (ipsilateral to the observed SOAE) LF exposure. The CAS consisted of a $90 \mathrm{~s}$, bandpass-filtered Gaussian noise $(100 \mathrm{~Hz}-8 \mathrm{kHz})$ presented at 65 or $70 \mathrm{~dB}$ SPL.

CAS is well known to suppress ipsilaterally recorded SOAEs during presentation and SOAEs quickly recover after CAS offset within less than $1 \mathrm{~s}$ (Zhao and Dhar 2010, 2011). Due to the duration of our analysis segments (5 s), the SOAEs already fully recovered from the CAS exposure within the first analysis segment. Consequently, we found no SOAEs fulfilling our criteria for the Bounce or indeed any other significant changes in the CAS control recordings. When the CAS was presented simultaneously with the ipsilateral LF tone, however, Bouncing of permanent SOAEs after LF offset changed significantly. Exemplary time courses of a preexisting and a transient SOAE are shown in Fig. 4a and b, respectively. While in the reference recording (red) the preexisting SOAE showed a significant biphasic Bounce, presentation of a $65 \mathrm{~dB}$ SPL (blue) or $70 \mathrm{~dB}$ SPL (green) CAS together with the ipsilateral LF tone clearly affected the magnitude of the Bounce.

Overall, temporary level reductions of preexisting SOAEs were less pronounced with CAS than without (Wilcoxon signed rank test, $p=0.085$ and 0.007 for CAS

a

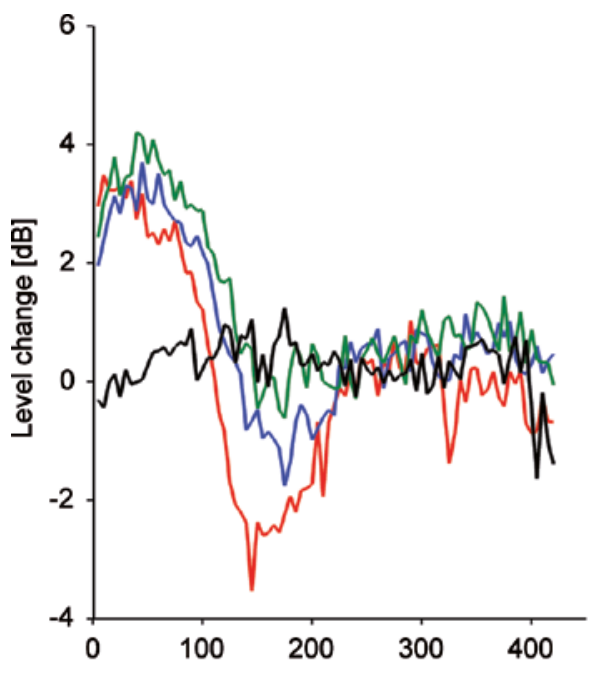

b

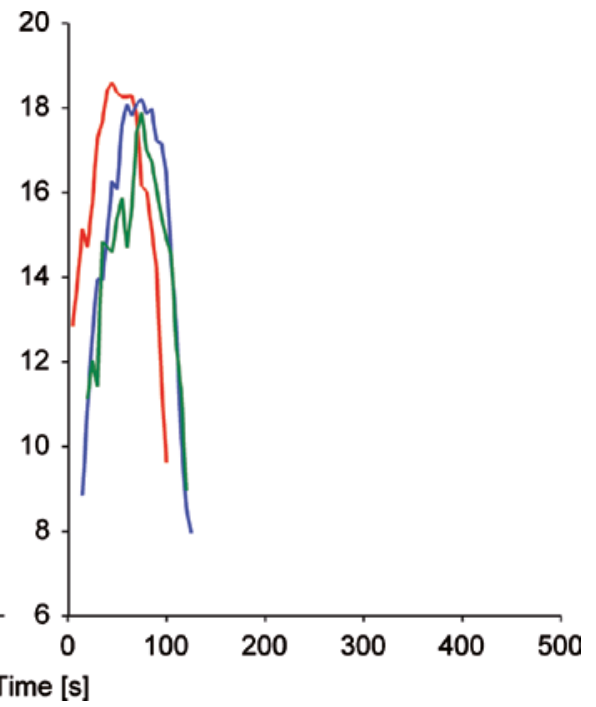

Fig. 4 A preexisting SOAE (a), and a new, transient SOAE (b) after LF exposure (red) and after LF exposure witch simultaneous CAS at $65 \mathrm{~dB}$ SPL (blue) or $70 \mathrm{~dB}$ SPL (green), and after CAS exposure alone (black) 
levels of 65 and $70 \mathrm{~dB}$, respectively), while the SOAE level increases remained fairly unchanged. This resulted in a less symmetrical shape of the SOAE Bounce compared to the reference recording. Consequently, the time constants of the fitted function with CAS shortened significantly.

\section{Discussion}

The current biophysical experiments reveal a significant effect of LF sound exposure on the inner ear, and specifically on the function of OHCs. The observed effects are reflected in significant changes of quadratic distortion products, but importantly, in changes of SOAEs, which (for the ears that exhibit them) allows for a leastinvasive evaluation of inner ear function.

As the low-frequency stimuli used in this study are intense, mechanisms associated with acoustic overexposure and recovery thereof could in principle be responsible for the Bounce phenomenon. Temporary or permanent damage to OHCs represent an unlikely cause, as recovery from acoustic overexposure with sounds in the sensitive range of hearing is typically monotonic, does not oscillate and no hypersensitivity can be seen. It has been shown, however, that intense sound stimulation of the cochlea in the isolated temporal bone preparation increases intracellular $\mathrm{Ca}^{2+}$ level of hair cells and supporting cells (Fridberger et al. 1998; Jacob et al. 2013). Acoustic injury consists of a plethora of structural and metabolic changes to the cochlea, with structural damage possibly masking more subtle (and possibly oscillating) metabolic changes of cochlear sensitivity caused by the rise of intracellular $\mathrm{Ca}^{2+}$ levels.

The observed effects support the hypothesis that $\mathrm{OHC} \mathrm{Ca}{ }^{2+}$ homeostasis is the source of the Bounce: Patuzzi (2011) suggested that the Bounce is a direct result of OHC activation by LF sound, rather than a secondary effect caused by modulation of stria vascularis activity, as the LF sound-induced endocochlear potential modulation during the Bounce (Kirk and Patuzzi 1997) could suggest. Patuzzi (2011) hypothesized that large, LF-induced receptor potentials in OHCs are the underlying cause of cochlear sensitivity oscillations associated with the Bounce phenomenon. These LF OHC receptor potentials are not attenuated by the low-pass characteristic of the OHC membrane. Patuzzi (2011) postulated that large, LF receptor potentials activate voltage-sensitive, $\mathrm{L}$-type $\mathrm{Ca}^{2+}$ channels in the $\mathrm{OHC}$ membrane. This results in an increase of intracellular $\mathrm{Ca}^{2+}$ in OHCs. $\mathrm{Ca}^{2+}$-induced $\mathrm{Ca}^{2+}$ release and -uptake mechanisms, with different time courses, can then cause slow, underdamped oscillations of $\mathrm{OHC}$ cytosolic $\mathrm{Ca}^{2+}$ concentrations, modulating the gain of the cochlear amplifier (Patuzzi 2011).

The increased damping (corresponding to decreased decay time constants) we observed in the CAS experiments indicates that processes re-adjusting the $\mathrm{Ca}^{2+}$ overshoot may accelerate due to the activation of the medial olivo-cochlear bundle by the CAS. Even while the LF sound is on, the LF-induced $\mathrm{Ca}^{2+}$ concentration changes presumably also undergo oscillations (Patuzzi 2011). In contrast, the slow 
efferent effect (Sridhar et al. 1997) can cause a constant $\mathrm{Ca}^{2+}$ release from OHC internal stores, the lateral cisternae, while CAS is on. We hypothesize that this constant influx of $\mathrm{Ca}^{2+}$ may help to accelerate $\mathrm{Ca}^{2+}$-dependent $\mathrm{Ca}^{2+}$-uptake and thus, a quicker recovery of the system.

In summary, the current experiments reveal a pronounced effect of LF exposure on the active mechanisms in the inner ear, as they are mediated by OHCs. Considering that the current LF exposure was limited to $90 \mathrm{~s}$, it is unclear how a longerduration LF exposure may affect the system.

Acknowledgments The authors wish to acknowledge the contributions of Margarete Überfuhr, David Laubender and Kathrin Kugler, who carried out the experimental procedures this chapter is based on.

This work was funded by a grant (EO 0901) from the German Ministry of Science and Education to the German Center for Vertigo and Balance Disorders (IFB), project TR-F9.

Open Access This chapter is distributed under the terms of the Creative Commons AttributionNoncommercial 2.5 License (http://creativecommons.org/licenses/by-nc/2.5/) which permits any noncommercial use, distribution, and reproduction in any medium, provided the original author(s) and source are credited.

The images or other third party material in this chapter are included in the work's Creative Commons license, unless indicated otherwise in the credit line; if such material is not included in the work's Creative Commons license and the respective action is not permitted by statutory regulation, users will need to obtain permission from the license holder to duplicate, adapt or reproduce the material.

\section{References}

Berglund B, Hassmen P, Job RF (1996) Sources and effects of low-frequency noise. J Acoust Soc Am 99(5):2985-3002

Burns EM (2009) Long-term stability of spontaneous otoacoustic emissions. J Acoust Soc Am 125(5):3166-3176. doi:10.1121/1.3097768

Dallos P (1986) Neurobiology of cochlear inner and outer hair cells: intracellular recordings. Hear Res 22:185-198

Dallos P, Santos-Sacchi J, Flock A (1982) Intracellular recordings from cochlear outer hair cells. Science 218(4572):582-584

Drexl M, Uberfuhr M, Weddell TD, Lukashkin AN, Wiegrebe L, Krause E, Gurkov R (2014) Multiple indices of the 'bounce' phenomenon obtained from the same human ears. J Assoc Res Otolaryngol 15(1):57-72. doi:10.1007/s10162-013-0424-x

Fex J (1967) Efferent inhibition in the cochlea related to hair-cell dc activity: study of postsynaptic activity of the crossed olivocochlear fibres in the cat. J Acoust Soc Am 41(3):666-675

Fridberger A, Flock A, Ulfendahl M, Flock B (1998) Acoustic overstimulation increases outer hair cell $\mathrm{Ca}^{2+}$ concentrations and causes dynamic contractions of the hearing organ. Proc Natl Acad Sci U S A 95(12):7127-7132

Guinan JJ (2012) How are inner hair cells stimulated? Evidence for multiple mechanical drives. Hear Res 292(1-2):35-50

Hirsh I, Ward W (1952) Recovery of the auditory threshold after strong acoustic stimulation. J Acoust Soc Am 24:131

Hughes JR (1954) Auditory Sensitization. J Acoust Soc Am 26(6):1064-1070. doi:10.1121/1.1907450 
Jacob S, Johansson C, Fridberger A (2013) Noise-induced alterations in cochlear mechanics, electromotility, and cochlear amplification. Pflügers Archiv—European. J Physiol 465(6):907917. doi:10.1007/s00424-012-1198-4

Kirk DL, Patuzzi RB (1997) Transient changes in cochlear potentials and DPOAEs after lowfrequency tones: the 'two-minute bounce' revisited. Hear Res 112(1-2):49-68

Kugler K, Wiegrebe L, Grothe B, Kössl M, Gürkov R, Krause E, Drexl M (2014). Low-frequency sound affects active micromechanics in the human inner ear. R Soc Open Sci 1(2):140-166

Nowotny M, Gummer AW (2006) Nanomechanics of the subtectorial space caused by electromechanics of cochlear outer hair cells. Proc Natl Acad Sci U S A 103(7):2120-2125. doi:10.1073/ pnas.0511125103

Patuzzi R (2011) Ion flow in cochlear hair cells and the regulation of hearing sensitivity. Hear Res 280(1-2):3-20. doi:10.1016/j.heares.2011.04.006

Salt AN, Hullar TE (2010) Responses of the ear to low frequency sounds, infrasound and wind turbines. Hear Res 268(1-2):12-21. doi:S0378-5955(10)00312-6 [pii] 10.1016/j. heares.2010.06.007

Salt AN, Lichtenhan JT, Gill RM, Hartsock JJ (2013) Large endolymphatic potentials from low-frequency and infrasonic tones in the guinea pig. J Acoust Soc Am 133(3):1561-1571. doi:10.1121/1.4789005

Sridhar TS, Brown MC, Sewell WF (1997) Unique postsynaptic signaling at the hair cell efferent synapse permits calcium to evoke changes on two time scales. J Neurosci 17(1):428-437

Zhao W, Dhar S (2010) The effect of contralateral acoustic stimulation on spontaneous otoacoustic emissions. J Assoc Res Otolaryngol 11(1):53-67. doi:10.1007/s10162-009-0189-4

Zhao W, Dhar S (2011) Fast and slow effects of medial olivocochlear efferent activity in humans. PloS ONE 6(4):e18725. doi:10.1371/journal.pone.0018725 